\title{
BMJ Open Impact of home telemonitoring and management support on blood pressure control in non-dialysis CKD: a systematic review protocol
}

Ikechi G Okpechi (D) , ${ }^{1,2}$ Shezel Muneer, ${ }^{2}$ Mohammed M Tinwala, ${ }^{2}$ Deenaz Zaidi, ${ }^{2}$ Laura N Hamonic, ${ }^{3}$ Branko Braam, ${ }^{4}$ Kailash Jindal, ${ }^{1}$ Scott Klarenbach, ${ }^{2}$ Raj S Padwal, ${ }^{5}$ Soroush Shojai, ${ }^{6}$ Stephanie Thompson (D) , ${ }^{1,7}$ Aminu K Bello ${ }^{8}$

To cite: Okpechi IG, Muneer S, Tinwala MM, et al. Impact of home telemonitoring and management support on blood pressure control in nondialysis CKD: a systematic review protocol. BMJ Open 2021;11:e044195. doi:10.1136/ bmjopen-2020-044195

- Prepublication history for this paper is available online. To view these files, please visit the journal online (http://dx.doi. org/10.1136/bmjopen-2020044195).

Received 26 August 2020 Accepted 08 May 2021
Check for updates

(C) Author(s) (or their employer(s)) 2021. Re-use permitted under CC BY-NC. No commercial re-use. See rights and permissions. Published by BMJ.

For numbered affiliations see end of article.

Correspondence to

Aminu K Bello;

aminu1@ualberta.ca

\section{ABSTRACT}

Introduction Hypertension is a common public health problem and a key modifiable risk factor for cardiovascular (CV) and chronic kidney disease (CKD). Home blood pressure (BP) telemonitoring (HBPT) and management is associated with improved BP control, accelerated delivery of care and decision-making strategies that can reduce adverse outcomes associated with hypertension. The aim of this paper is to describe the protocol for a systematic review to assess the impact of HBPT interventions used for improving BP control and reducing CV and kidney outcomes in non-dialysis CKD patients.

Methods We developed this protocol using the Preferred Reporting Items for Systematic reviews and Meta-Analyses for Protocols 2015. We will search empirical databases such as MEDLINE, Embase, Cochrane Library, CINAHL, Web of Science and PsycINFO and grey literature for studies conducted in non-dialysis CKD patients on interventions using HBPT and reporting outcomes related to BP control and other outcomes such as CV events and kidney disease progression. All studies meeting these criteria, in adults and published from inception until 2020 with no language barrier will be included.

Ethics and dissemination Ethical approval will not be required for this review as the data used will be extracted from already published studies with publicly accessible data. As this study will assess the impact of HBPT on BP control in non-dialysis CKD patients, evidence gathered through it will be disseminated using traditional approaches that includes open-access peer-reviewed publication, scientific presentations and a report. We will also disseminate our findings to appropriate government agencies.

PROSPERO registration number CRD42020190705).

\section{INTRODUCTION}

Hypertension, also known as raised or high blood pressure (BP), is a prevalent global public health problem and an important modifiable risk factor for cardiovascular disease (CVD) and chronic kidney disease (CKD). Hypertension is defined as office systolic BP (SBP) values $\geq 140 \mathrm{~mm} \mathrm{Hg}$ and/or diastolic
Strengths and limitations of this study

- This study will assess the impact of home blood pressure telemonitoring on cardiovascular (CV) and kidney-related outcomes in non-dialysis chronic kidney disease (CKD) patients.

- Focus on non-dialysis CKD population is to reduce biases induced by recurrent haemodynamic changes with salt retention and volume status in CKD patients receiving dialysis, and lack of a standardised BP target in patients on dialysis.

- The key outcomes of interest include changes in blood pressure control, progression of CKD (estimated glomerular filtration rate, proteinuria criteria), hospitalisations, incident fatal and non-fatal CV events, all-cause mortality, cost effectiveness, patient-reported outcome measures and patientreported experience measures.

- We will assess the quality of studies using a tool that incorporates assessments of risk of bias across core study domains: sampling, sampling technique and size, outcome measurement, response rate and statistical reporting.

- A potential limitation of this study could be heterogeneity and number of studies of low quality which could affect pooled estimates and our ability to conduct a meta-analysis.

BP (DBP) values $\geq 90 \mathrm{~mm} \mathrm{Hg}$ (table 1$).{ }^{1}$ The prevalence of hypertension in the global adult population was estimated to be $31.1 \%$ (95\% CI: $30.0 \%$ to $32.2 \%$ ) in 2010 , representing 1.38 billion people who were affected worldwide. ${ }^{2}$ Notwithstanding the extensive availability of effective treatment options, BP control remains suboptimal, especially in low-income and middle-income countries for reasons that includes poor-adherence, clinical inertia and organisational failure. ${ }^{2}{ }^{3} \mathrm{~A}$ number of interventions have been targeted at improving medications adherence, as it is a major reason for poor BP control, including 
Table 1 Definition and classification of hypertension (ESH)

\begin{tabular}{|c|c|c|c|}
\hline Category & SBP (mm Hg) & & DBP $(\mathrm{mm} \mathrm{Hg})$ \\
\hline Optimal & $<120$ & and & $<80$ \\
\hline Normal & $120-129$ & and/or & $80-84$ \\
\hline High normal & $130-139$ & and/or & $85-89$ \\
\hline $\begin{array}{l}\text { Grade } 1 \\
\text { hypertension }\end{array}$ & $140-159$ & and/or & 90-99 \\
\hline $\begin{array}{l}\text { Grade } 2 \\
\text { hypertension }\end{array}$ & $160-179$ & and/or & $100-109$ \\
\hline $\begin{array}{l}\text { Grade } 3 \\
\text { hypertension }\end{array}$ & $\geq 180$ & and/or & $\geq 110$ \\
\hline $\begin{array}{l}\text { Isolated systolic } \\
\text { hypertension }\end{array}$ & $\geq 140$ & and & $<90$ \\
\hline Office BP & $\geq 140$ & and/or & $\geq 90$ \\
\hline \multicolumn{4}{|l|}{ Ambulatory BP } \\
\hline $\begin{array}{l}\text { Daytime (or } \\
\text { awake) mean }\end{array}$ & $\geq 135$ & and/or & $\geq 85$ \\
\hline $\begin{array}{l}\text { Night-time (or } \\
\text { asleep) mean }\end{array}$ & $\geq 120$ & and/or & $\geq 70$ \\
\hline 24-hour mean & $\geq 130$ & and/or & $\geq 80$ \\
\hline Home BP mean & $\geq 135$ & and/or & $\geq 85$ \\
\hline
\end{tabular}

BP, blood pressure; DBP, diastolic blood pressure; ESH, European Society of Hypertension; SBP, systolic blood pressure.

those at physician level (eg, improving counselling and education), patient level (eg, self-monitoring of BP) and at healthcare system level (eg, support to the development of monitoring systems). ${ }^{1}$

Some of the major challenges with care in hypertension relates to the proportion of people who are aware (diagnosed), receiving treatment or those treated who have achieved control to target of their BP. Data from the International Society of Hypertension (ISH) screening programme (May Measurement Month (MMM)) in 2019 showed that of 1.5 million people who were screened for hypertension, $32.0 \%$ had never had a BP measurement before and $34.0 \%$ had hypertension. Of those identified to be hypertensives, $58.7 \%$ were aware, $54.7 \%$ were on treatment, $31.7 \%$ were controlled to $<140 / 90 \mathrm{~mm} \mathrm{Hg}$ and $23.3 \%$ had untreated or inadequately treated hypertension. ${ }^{4}$ The results of previous ISH regional screening programmes for MMM support this global trend. ${ }^{56}$ The low proportion of patients with hypertension who are controlled suggests a need for practical and sustainable models to improve BP control at the population level in order to reduce the excess risk of CVD and other target organ damage associated with hypertension.

\section{Hypertension in CKD}

Hypertension is a common cause of CKD and highly prevalent among patients with CKD with an increased incidence and prevalence as kidney function declines. Hypertension is present in as high as $87.5 \%$ of CKD patients compared with only $28.5 \%$ of patients in the general population. ${ }^{7}$
The United States Renal Data System reports that hypertension is present in about $23.3 \%$ of the general population without CKD and in patients with CKD, occurs in $35.8 \%$ (stage 1), $48.1 \%$ (stage 2), $59.9 \%$ (stage 3) and $84.1 \%$ (stages 4 and 5). ${ }^{8}$ Guideline recommendations for diagnosing, monitoring and treating hypertension in the general population and in patients with CKD are frequently revised and updated. ${ }^{1910}$ The Kidney Disease Improving Global Outcomes (KDIGO) guideline on management of $\mathrm{BP}$ in CKD recommends the use of lifestyle modifications and pharmacological treatments for lowering $\mathrm{BP}$ in non-dialysis CKD patients. These measures include individualising BP targets with the use of various BP lowering agents, achieving and maintaining a healthy weight (BMI: $20-25 \mathrm{~kg} / \mathrm{m}^{2}$ ), lowering salt intake to $<2 \mathrm{~g}$ $(<90 \mathrm{mmol}$ of sodium) per day, undertaking exercise that is compatible with $\mathrm{CV}$ health and tolerance for at least 30 min five times per week and limiting intake of alcohol as options for BP control. ${ }^{9}$

BP exhibits a high level of short-term (24-hour ambulatory recordings) and long-term (office visit-to-visit) variability and both are associated with adverse outcomes independent of mean 24-hour or office-to-office BP values. ${ }^{1112}$ A number of studies have reported on the association between $\mathrm{BP}$ variability and risk $\mathrm{CV}$ events, progression of kidney failure or death in patients with CKD. ${ }^{13-15}$ Although they mainly report no usefulness of short-term variability in predicting adverse events in CKD patients, they show an association with CV events and death using long-term BP variability. In one Italian study of 402 CKD patients with median follow-up of 4.8 years, although long-term BP variability was associated with composite end-point of CV event or death (HR: 1.24; 95\% CI: 1.01 to 1.51 per $5 \mathrm{~mm} \mathrm{Hg}$ higher systolic difference of office SBP), short-term SBP variability was not (HR: 0.92; 95\% CI: 0.68 to 1.25 per $5 \mathrm{~mm} \mathrm{Hg}$ higher SD of 24-hour ambulatory systolic BP). ${ }^{13}$ In another large populationbased cohort that included 225759 Chinese hypertensive adults with median follow-up of 70.5 months, there were 25714 CV events, 27603 incident CKD and 16778 deaths reported. SBP variability was continuously and positively associated with increased CV events (HR: 1.35, 95\% CI: 1.30 to 1.39 ), incident CKD (HR: $1.39,95 \%$ CI: 1.35 to 1.43 ) and mortality risk (HR: $1.40,95 \%$ CI: 1.34 to 1.45$){ }^{16}$

\section{Home blood pressure telemonitoring (HBPT)}

$\mathrm{BP}$ recorded out-of-office (either home BP monitoring (HBPM) or ambulatory BP monitoring (ABPM)) provide readings taken in conditions that are more representative of daily life than conventional office readings. Given that the goal of hypertension detection and treatment is to reduce mortality, and adverse $\mathrm{CV}$ and kidney outcomes, use of HBPM is encouraged as it is more accurate and superior to office BP monitoring (OBPM) in predicting CV events and all-cause mortality. ${ }^{17} 18$ Also, OBPM does not always correctly identify patients with hypertension due to 'white-coat' or 'masking' effects, however, HBPM improves BP monitoring and provides more representative 
BP data and better prediction of outcomes. ${ }^{19}$ The ability to transmit, in real-time, data from HBPM device to a caregiver improve the chance of better BP control when combined to decision-making strategies can reduce adverse outcomes associated with hypertension. ${ }^{20}$

Home BP telemonitoring (HBPT) is based on the use of clinically validated electronic automated BP monitors storing $\mathrm{BP}$ values obtained at patient's home and promotes a more effective link between patients and their caregivers. ${ }^{20}{ }^{21}$ Increasingly, researchers have leveraged on telemonitoring technology for the monitoring and treatment of patients with various chronic conditions such as heart diseases ${ }^{22}$ respiratory diseases, ${ }^{23}$ diabetes ${ }^{24}$ and hypertension. ${ }^{25} 26$

The Telemonitoring and Self-Management in Hypertensions (TASMINH2) study has shown that self-management of hypertension is possible as most participants made at least one medication change, were confident about selfmonitoring and many felt their multiple home readings were more valid than single office readings taken by their doctor. ${ }^{27}{ }^{28}$ In a subsequent study (TASMINH4), when compared with usual care, the adjusted mean SBP differences with self-monitoring was $-3.5 \mathrm{~mm} \mathrm{Hg}$ (95\% CI: -5.8 to $-1.2 ; \mathrm{p}=0.0029)$ and $-4.7 \mathrm{~mm} \mathrm{Hg}(-7.0$ to $-2.4 ; \mathrm{p}<0.0001)$ ) for telemonitoring. ${ }^{29}$ HBPT has also been shown to be cost-effective ${ }^{30}$ and more effective in achieving BP control than usual care (RR: 1.16; 95\% CI: 1.08 to $1.25 ; \mathrm{p}<0.001) .{ }^{31}$ However, when HBPT was combined with additional care (eg, counselling, education, behavioural management, etc.) and compared with HBPT alone, there were increased mean changes in SBP and DBP, suggesting that HBPT can be more efficacious when proactive additional support is provided. ${ }^{31}$

Other outcomes (eg, quality of life, QoL and cost) have also been evaluated. For example, in patients with kidney disease, telemonitoring has also been shown as a useful tool for improving QoL ${ }^{32}$ and associated with reduced healthcare resource utilisation and costs in patients receiving automated peritoneal dialysis. ${ }^{33} \mathrm{~A}$ recent systematic review and meta-analysis was conducted to evaluate the effects of telehealth on BP management in non-dialysis CKD patients. ${ }^{34}$ From the two studies they included for meta-analysis, pooled estimates showed decreased SBP (mean difference (MD), -5.10 ; 95\% CI: -11.34 to 1.14 ; $\mathrm{p}=0.11$ ), increased DBP (MD: $0.45 ; 95 \%$ CI: -4.24 to 5.13 ; $\mathrm{p}=0.85$ ), decreased serum creatinine (pooled MD: -0.38 ; 95\% CI: -0.83 to $0.07 ; \mathrm{p}=0.10$ ) and maintained estimated glomerular filtration rate (eGFR) (pooled MD: 4.72; $95 \% \mathrm{CI}:-1.85$ to $11.29 ; \mathrm{p}=0.16$ ) in the telehealth group. However, Luo et $a l^{34}$ used studies with telehealth interventions for BP control in only stages 3-5 CKD patients. Table 2 is a summary of the characteristics of their study design and the planned characteristics of our study.

\section{Objective}

Given that an increasing number of studies ${ }^{25-29}$ have shown the efficacy of HBPT on hypertension control and outcomes with dearth of data for CKD, the aim of the current review is to specifically determine the impact of HBPT and management support on BP control and other prespecified CV and kidney-related outcomes in patients with non-dialysis CKD.

\section{METHODS AND ANALYSIS}

We used the Preferred Reporting Items for Systematic reviews and Meta-Analyses for Protocols 2015 (PRISMA-P 2015) to develop this protocol. ${ }^{35}$

\section{Criteria for considering studies for the review \\ Types of studies}

We plan to include all study designs including time series studies, before/after studies, observational studies, randomised controlled trials (RCTs) as well previously published reviews that evaluated telemonitoring for BP control or reports an outcome.

\section{Types of participants}

We will include studies that have participants over 18 years of age, regardless of sex and ethnicity with a diagnosis of CKD (stages 1-5, but not on dialysis and not transplanted).

\section{Types of interventions}

The intervention of interest will be the use of HBPT (with or without management support-nurses, pharmacist, physician, informed self-management of medications, health aids, exercise programmes, nutritional programmes, etc.) for BP assessment and monitoring. A telemonitoring intervention will be defined as any process or programme that involves transmission of $\mathrm{BP}$ records via information and communication technologies (ICT) using conduits leveraging a telephone or internet line (phones, computers, tablets, etc.). To be eligible, included studies will have reported on at least one outcome of interest. Comparators will include usual care and other interventions such as other BP device, education, counselling and behavioural management used to control BP. Studies that include only patients with CKD and no comparators will also be included if they meet other inclusion criteria.

\section{Types of outcome assessments}

The primary outcome will be any changes in mean SBP, mean DBP and/or mean arterial pressure (MAP) as well as proportion of controlled BP defined by each randomised trial's investigators. Secondary outcomes will include progression of CKD (eGFR, proteinuria criteria), hospitalisations, incident fatal and non-fatal CV events, all-cause mortality, cost effectiveness, patient-reported outcome measures and patient-reported experience measures.

\section{Search methods for identification of studies}

Electronic searches

We will electronically search the following databases: MEDLINE, Embase, Cochrane Library, CINAHL, ISI 


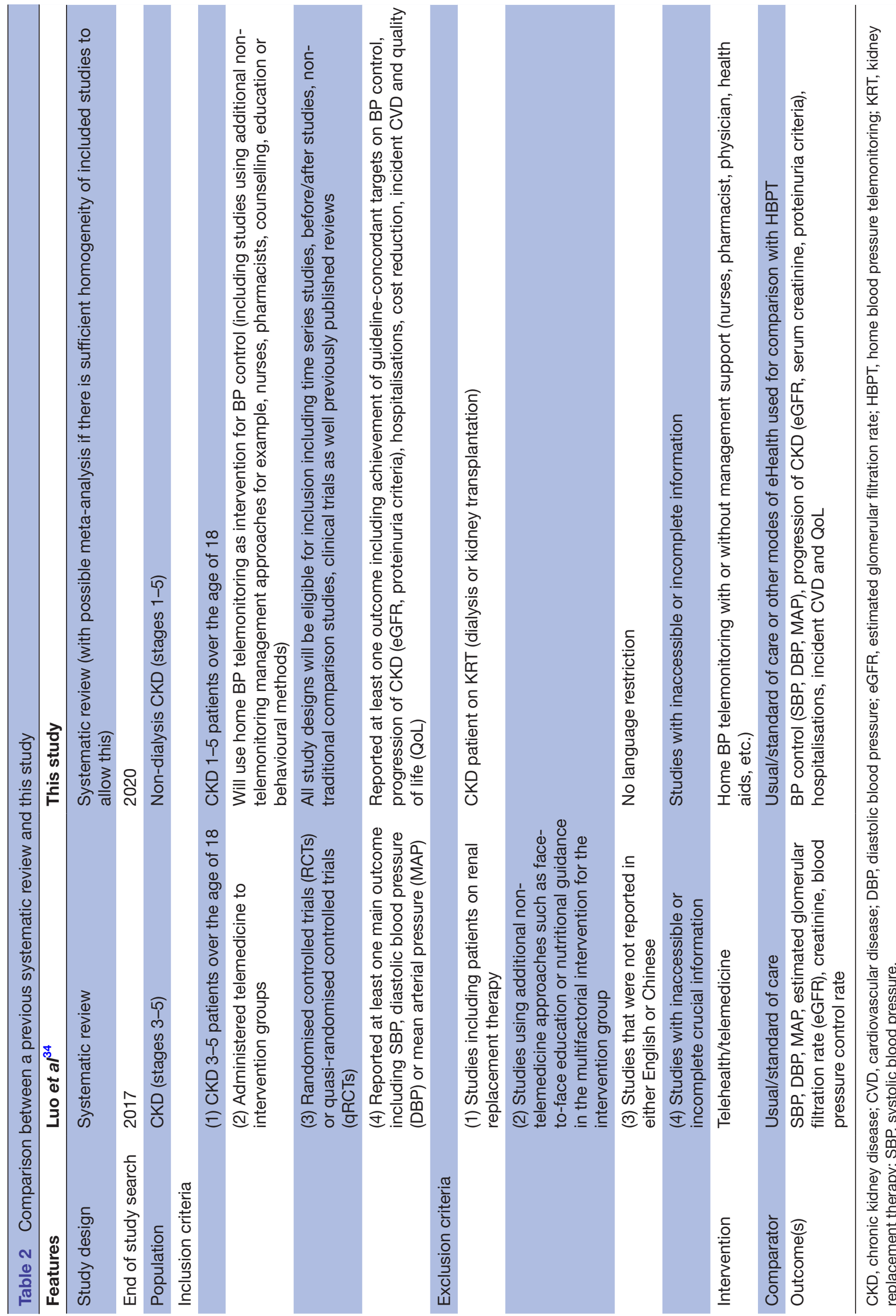

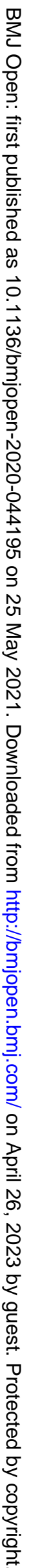


Web of Science and PsycINFO. We will search for studies of interventions published from inception to 2020 with no language restriction and designed to compare the impact of telemonitoring of BP with management support (nurses, pharmacist, physician, health aids, etc.) compared with usual care in improving BP control and other outcomes in non-dialysis CKD patients. The search strategy will be developed after discussion among reviewers using guidance from the Cochrane handbook. ${ }^{36}$ Using controlled vocabulary, we will adapt the MEDLINE search strategy for other databases. The search strategy for MEDLINE is shown in table 3.

\section{Other sources}

We will search the bibliographies of all relevant and selected publications for further studies and will also search grey literature using recommended resources in consultation with our medical Librarian. Thus, we will search ProQuest Dissertations and Theses Global, and Conference Proceedings Citation Index (Clarivate Analytics).

\section{Data collection and analysis \\ Study selection}

We will use a two-stage collaborative review process for screening and selection of studies to be included. In the first stage, two reviewers (SM and MMT) will independently assess the titles/abstracts of retrieved studies to be selected for full-text screening if conducted in a nondialysis CKD population (stages 1-5). In the second stage, full texts, having met the above criteria will be obtained for further screening and will be included if HBPT (with or without management support-nurses, pharmacist, physician, health aids, etc.) is used as the intervention and the study reports one of the stated outcomes of interest. A third reviewer (IGO) will evaluate any discrepancies, if necessary, and will advise in case of disagreement. We will record all reasons for exclusion and exclude studies not using HBPT as the intervention to improve BP control. Figure 1 is a summary of the process that will be used for study selection. Thus, the inclusion and exclusion criteria for the study will be:

\section{Inclusion criteria}

- Studies conducted in a non-dialysis CKD population.

- Studies using HBPT (with or without management support, ie, nurses, pharmacist, physician, health aids, etc.) as the intervention.

- Studies reporting on at least one outcome measure (BP change/control, CV outcomes or CKD outcomes, patient-reported outcome measures and patientreported experience measures).

- Studies that include only patients with CKD and no comparators will be included if they meet other inclusion criteria.

- Publication date (no restriction).

- Language restriction (none).

\section{Exclusion criteria}

- Studies reporting other forms of ehealth for hypertension control but not involving BP telemonitoring.

- Review articles, editorials, letters to the editor, commentaries, case studies, case reports, images and studies in which we are unable to get relevant data even after attempts to get these from the authors.

- Studies in which the specific outcomes of interest cannot be clearly identified or extrapolated (eg, studies reporting differences between groups but not providing information on the entire group).

\section{Data extraction and management}

Two reviewers (SM and MMT) will independently extract data and summarise the details of selected studies using a standard data extraction sheet. All extracted data will be reviewed for accuracy and completeness. The data items we will collect will include general study characteristics (eg, study type, publication year country, etc.), study design (RCT, observational, case-control study, cohort, etc.), type of intervention utilised (HBPT alone or with management support), duration of intervention, outcomes and conclusions. If more than one outcome time (eg, 12 and 24 months) is reported, the data on the longest follow-up will be extracted.

\section{Assessment of risk of bias in included studies}

Methodological quality will be evaluated using the checklist developed by Hoy $e t a l^{87}$ to assess the risk of bias in primary studies. This quality assessment tool incorporates assessments of risk of bias across core domains including sampling, the sampling technique and size, outcome measurement, response rate and statistical reporting. We will also present the overall risk of bias per study in a risk of bias summary table and we will examine for publication bias using a funnel plot. If the funnel plot is asymmetrical, we will explore possible causes including publication bias, poor methodological quality and true heterogeneity.

\section{Measures of treatment effect}

We will present the effects on BP between interventions at follow-up (SBP and DBP) according to the HBPT interventions proposed in each study. Dichotomous outcomes will be presented as risk ratios, while continuous outcomes will be presented as MD between the change in the intervention and control groups if the outcomes have been measured and reported in the same way across all studies. If the continuous outcomes have been measured in different ways across studies, then we will use the standardised MD between the intervention and control groups. We will present time-to-event outcomes as HR. We will report $95 \%$ CIs for all outcomes.

\section{Dealing with missing data}

In the case of missing or unclear data, we will contact the authors to request such information related to study methods, attrition rates and outcomes. Where possible, we will calculate missing data using available relevant information including imputing data, where appropriate. 


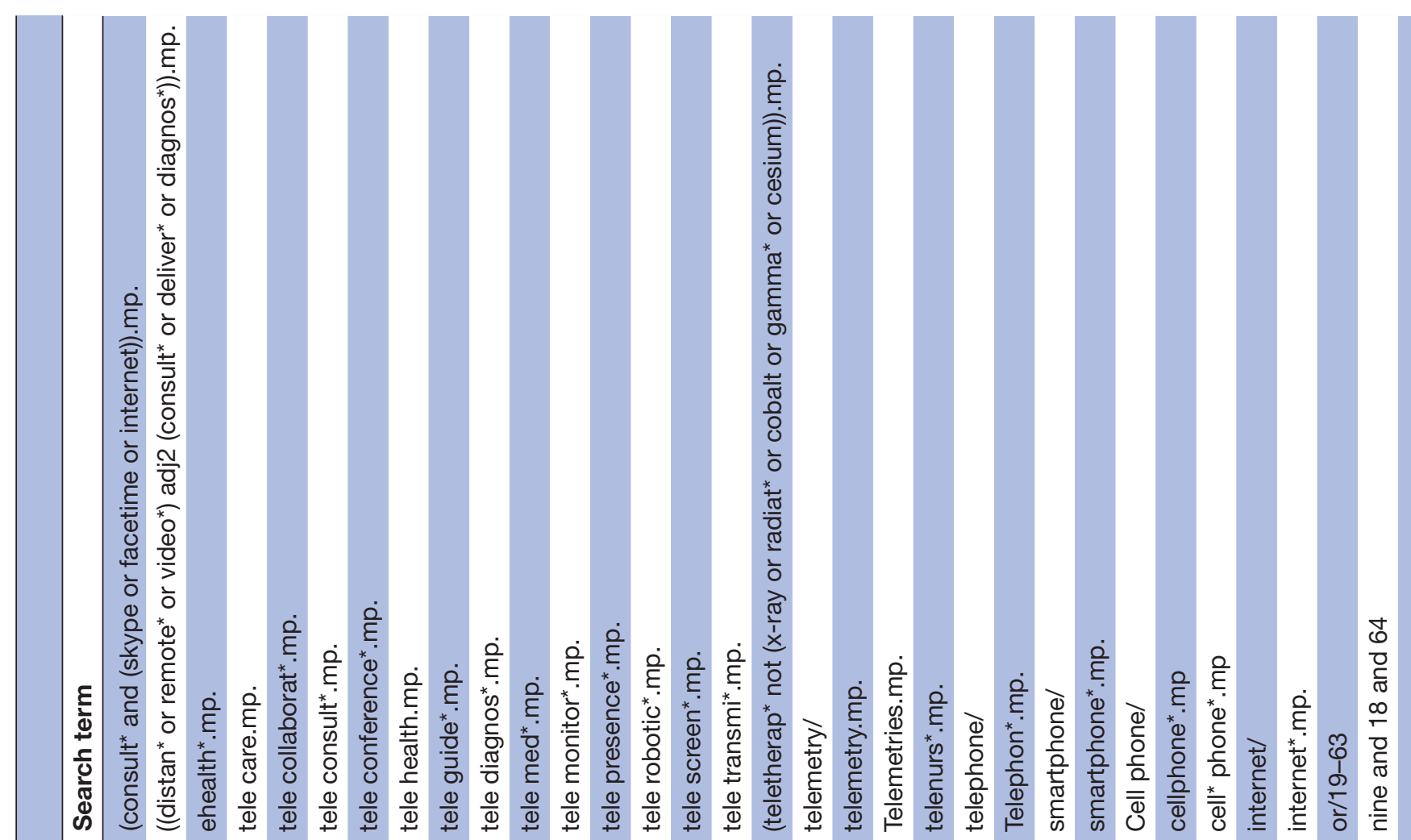

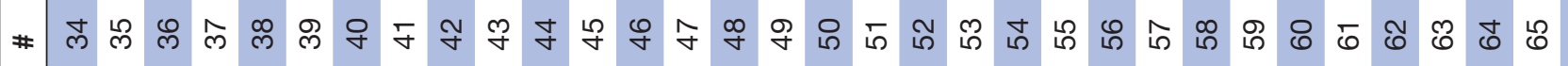

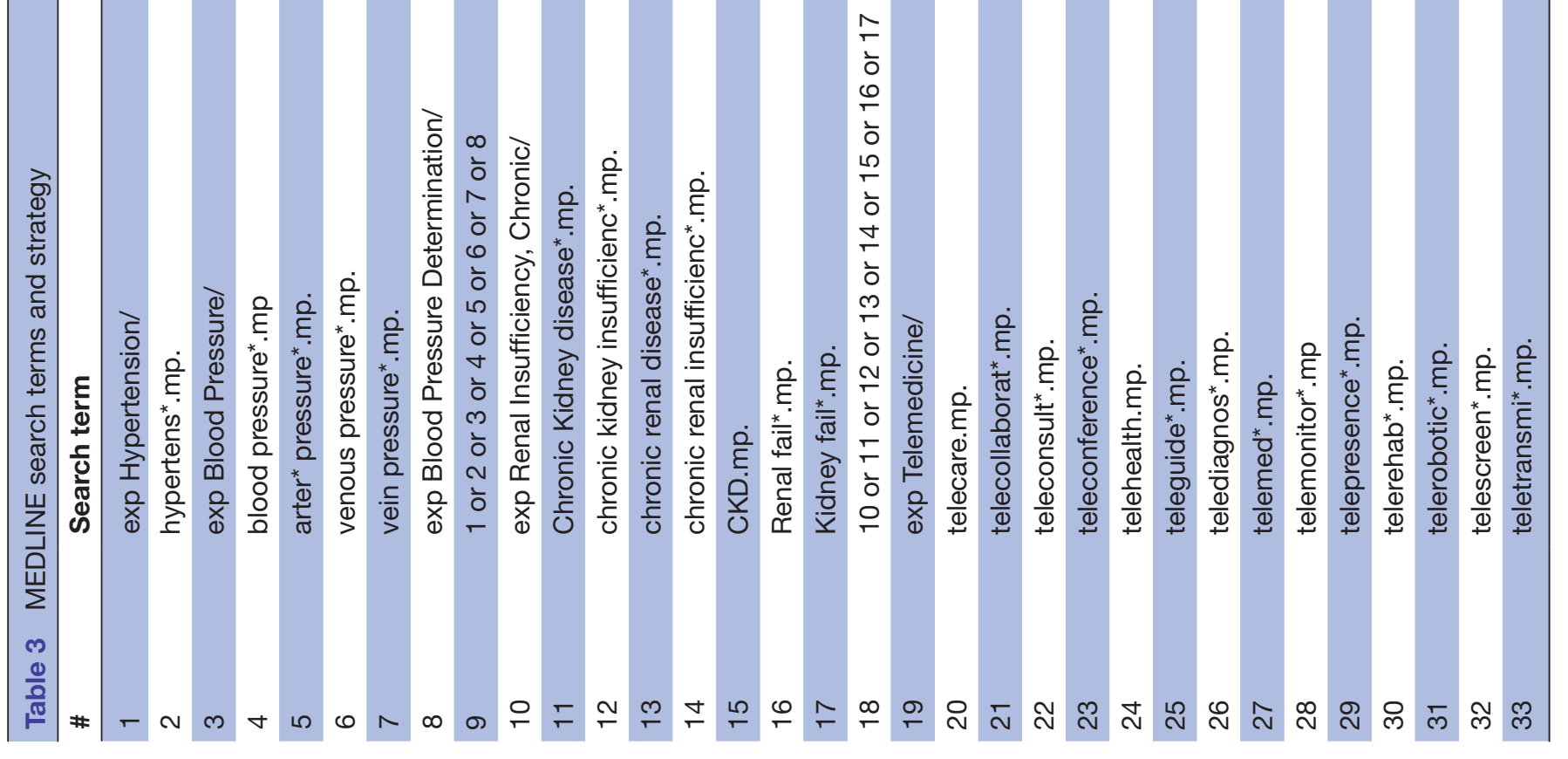




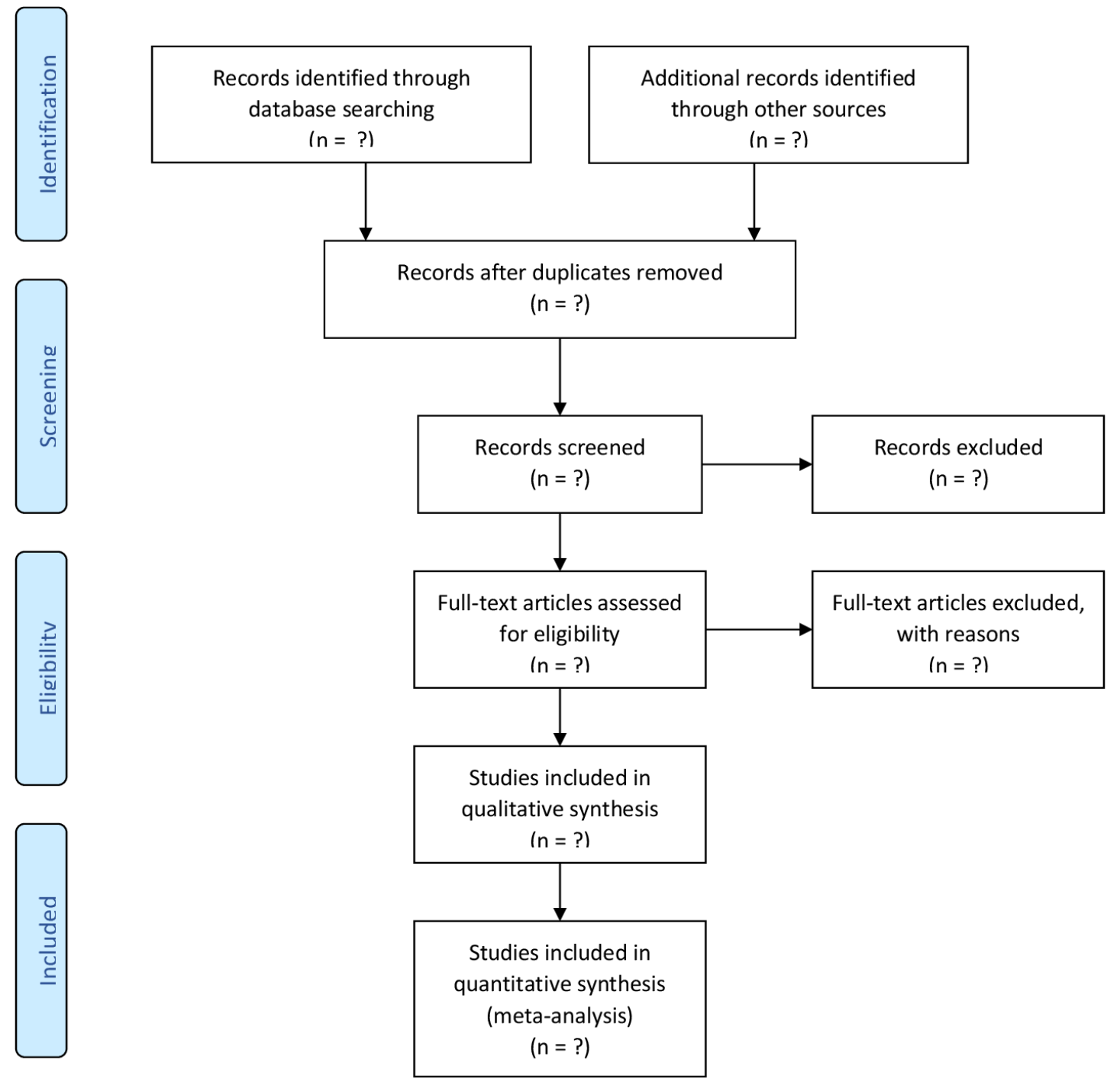

Figure 1 PRISMA flow chart for process of study selection. PRISMA, Preferred Reporting Items for Systematic Reviews and Meta-Analyses.

All missing outcome data will also be reported in the data extraction form and risk of bias table.

\section{Assessment of heterogeneity}

We will assess heterogeneity among studies in relation to participant characteristics (diabetic CKD and others), intervention type (HBPT alone or HBPT plus management), duration and outcome (BP control, CKD progression, death or QoL). We will test statistical heterogeneity using the $\chi^{2}$ test (considering a value of $p<0.1$ to indicate heterogeneity) and estimate the amount of heterogeneity using the $\mathrm{I}^{2}$ statistic ( $\mathrm{I}^{2}$ values of $<25 \%, 25 \%-50 \%$ and $>50 \%$ represent low, medium and high heterogeneity, respectively). ${ }^{36}$ We will assess reasons for heterogeneity through subgroup analysis.

\section{Data synthesis}

We will summarise the characteristics of included studies in a table and we will assess if there is possibility to conduct a meta-analysis. If the characteristics of included studies are excessively heterogeneous, we will not pool results, but we will only present a narrative synthesis of the results of group findings by context measures. If a meta-analysis is conducted, intervention effects will be calculated as relative risks (RR) with $95 \%$ CIs for dichotomous data and we will calculate MD with $95 \%$ CIs for continuous variables. Whether a fixed-effects model or a random-effects model will be used depends on the results of the $\chi^{2}$ test and $\mathrm{I}^{2}$ test for heterogeneity. If there is substantial statistical heterogeneity, we will adopt a random-effects model, whereas a fixed-effects model will be used if there is no substantial statistical heterogeneity $\left(\mathrm{I}^{2}<50 \%\right)$.

\section{Subgroup analysis}

Subgroup analysis will be considered according to the following variables: age, gender, CKD stage, study setting (rural vs urban or low-income and middle-income vs highincome using the World Bank country classifications by income level $)^{38}$ study duration ( $<6$ months vs $>6$ months) and hypertension status (controlled vs uncontrolled). 


\section{Patient and public involvement}

Patients and the public will not be involved in this study.

\section{Ethics and dissemination}

Ethical approval will not be needed for this study as data used will be extracted from already published studies. Our dissemination strategy will use traditional approaches, including open-access peer-reviewed publication(s), scientific presentations and a report.

\section{DISCUSSION}

Hypertension is the leading prognostic marker for risk of adverse health outcomes in patients with CKD, and effective $\mathrm{BP}$ control to mitigate this risk remains a challenge. There is limited data on the use of HBPT for assessing and monitoring BP control in patients with CKD. This work will therefore provide new information on the potential role of HBPT in the management of hypertension and reducing adverse health outcomes in comparison with usual care. As telehealth practices and telemonitoring technologies continue to evolve worldwide, this study will demonstrate the impact of HBPT for hypertension monitoring and control as well as its impact on fatal and nonfatal CV events, progression of kidney function, QoL and death in non-dialysis CKD patients. Strengths and limitations of this study will be highlighted in the process of identified evidence.

\section{Author affiliations}

${ }^{1}$ Division of Nephrology and Hypertension, University of Cape Town, Cape Town, South Africa

${ }^{2}$ Division of Nephrology and Department of Medicine, University of Alberta, Edmonton, Alberta, Canada

${ }^{3}$ John W. Scott Health Sciences Library, University of Alberta, Edmonton, Alberta, Canada

${ }^{4}$ Nephrology, University of Alberta Faculty of Medicine and Dentistry, Edmonton, Alberta, Canada

${ }^{5}$ Department of Medicine, University of Alberta, Edmonton, Alberta, Canada ${ }^{6}$ University of Alberta Faculty of Medicine and Dentistry, Edmonton, Alberta, Canada ${ }^{7}$ Nephrology, University of Alberta, Edmonton, Alberta, Canada

${ }^{8}$ Medicine, University of Alberta Faculty of Medicine and Dentistry, Edmonton, Alberta, Canada

\section{Twitter Stephanie Thompson @StephanieTh11}

Contributors IGO and AKB conceived the study design. The first version of the protocol was drafted by IGO and AKB, and was revised by SM, MMT, DZ, LNH, BB, KJ, SK, RSP, SS and ST. The search strategy was developed and performed by LNH. SM, MMT and DZ will perform the screening, study selection and collect data from all included studies. All authors drafted and critically reviewed this manuscript and approved the final version

Funding Funding for this study was provided through the Alberta Kidney Care- North (formerly, Northern Alberta Renal Program)/AMGEN incorporated Grant: Improving access to care for rural northern CKD patients through eHealth technologies. 'Award/Grant number is not applicable'.

Competing interests RSP is CEO of $\mathrm{mmHg}$, a digital health company creating guideline-concordant innovations to improve the efficiency of remote patient monitoring. All other authors declare no conflict of interest.

Patient and public involvement Patients and/or the public were not involved in the design, or conduct, or reporting or dissemination plans of this research.

Patient consent for publication Not required.

Provenance and peer review Not commissioned; externally peer reviewed.
Open access This is an open access article distributed in accordance with the Creative Commons Attribution Non Commercial (CC BY-NC 4.0) license, which permits others to distribute, remix, adapt, build upon this work non-commercially, and license their derivative works on different terms, provided the original work is properly cited, appropriate credit is given, any changes made indicated, and the use is non-commercial. See: http://creativecommons.org/licenses/by-nc/4.0/.

ORCID iDs

Ikechi G Okpechi http://orcid.org/0000-0002-6545-9715

Stephanie Thompson http://orcid.org/0000-0003-3109-6837

\section{REFERENCES}

1 Williams B, Mancia G, Spiering W, et al. 2018 practice guidelines for the management of arterial hypertension of the European Society of hypertension and the European Society of cardiology: ESH/ESC Task force for the management of arterial hypertension. $J$ Hypertens 2018;36:2284-309.

2 Mills KT, Bundy JD, Kelly TN, et al. Global disparities of hypertension prevalence and control: a systematic analysis of population-based studies from 90 countries. Circulation 2016;134:441-50.

3 Glynn LG, Murphy AW, Smith SM, et al. Interventions used to improve control of blood pressure in patients with hypertension. Cochrane Database Syst Rev 2010;3:Cd005182.

4 Beaney T, Schutte AE, Stergiou GS, et al. May measurement month 2019: the global blood pressure screening campaign of the International Society of hypertension. Hypertension 2020;76:333-41.

5 Molinero A, Ruilope LM, Tous S, et al. May measurement month 2017: an analysis of blood pressure screening in Spain-Europe. Eur Heart J Suppl 2019;21:D107-10.

6 Woodiwiss AJ, Kruger R, Norton GR, et al. May measurement month 2017: an analysis of blood pressure screening results in South AfricaSub-Saharan Africa. Eur Heart J Suppl 2019;21:D104-6.

7 Muntner $\mathrm{P}$, Anderson A, Charleston J, et al. Hypertension awareness, treatment, and control in adults with CKD: results from the chronic renal insufficiency cohort (CRIC) study. Am J Kidney Dis 2010;55:441-51.

8 USRDS. USRDS 2010 annual data report: atlas of chronic kidney disease and end-stage renal disease in the United States, National Institutes of health, National Institute of diabetes and digestive and kidney diseases, Bethesda, MD, 2010.

$9 \mathrm{KDIGO}$. Chapter 2: lifestyle and pharmacological treatments for lowering blood pressure in CKD Nd patients. Kidney Int Suppl 2012;2:347-56.

10 Whelton PK, Carey RM, Aronow WS, et al. 2017 ACC/AHA/AAPA ABC/ACPM/AGS/APhA/ASH/ASPC/NMA/PCNA guideline for the prevention, detection, evaluation, and management of high blood pressure in adults: Executive summary: a report of the American College of Cardiology/American heart association Task force on clinical practice guidelines. Hypertension 2018;71:1269-324.

11 Muntner P, Shimbo D, Tonelli M, et al. The relationship between visitto-visit variability in systolic blood pressure and all-cause mortality in the general population: findings from NHANES III, 1988 to 1994. Hypertension 2011;57:160-6.

12 Parati G, Ochoa JE, Lombardi C, et al. Blood pressure variability: assessment, predictive value, and potential as a therapeutic target. Curr Hypertens Rep 2015;17:537.

13 Mallamaci F, Tripepi G, D'Arrigo G, et al. Blood pressure variability, mortality, and cardiovascular outcomes in CKD patients. Clin J Am Soc Nephrol 2019;14:233-40.

14 Whittle J, Lynch Al, Tanner RM, et al. Visit-to-Visit variability of bp and CKD outcomes: results from the ALLHAT. Clin J Am Soc Nephrol 2016;11:471-80.

15 Borrelli S, Garofalo C, Mallamaci F, et al. Short-Term blood pressure variability in nondialysis chronic kidney disease patients: correlates and prognostic role on the progression of renal disease. $J$ Hypertens 2018;36:2398-405.

16 Wan EYF, Yu EYT, Chin WY, et al. Association of visit-to-visit variability of systolic blood pressure with cardiovascular disease, chronic kidney disease and mortality in patients with hypertension. $J$ Hypertens 2020;38:943-53.

17 Sega R, Facchetti R, Bombelli M, et al. Prognostic value of ambulatory and home blood pressures compared with office blood pressure in the general population: follow-up results from the Pressioni Arteriose Monitorate E Loro Associazioni (PAMELA) study. Circulation 2005;111:1777-83.

18 Ohkubo T, Imai Y, Tsuji I, et al. Home blood pressure measurement has a stronger predictive power for mortality than does screening 
blood pressure measurement: a population-based observation in Ohasama, Japan. J Hypertens 1998;16:971-5.

19 Ward AM, Takahashi O, Stevens R, et al. Home measurement of blood pressure and cardiovascular disease: systematic review and meta-analysis of prospective studies. J Hypertens 2012;30:449-56.

20 Omboni S, Ferrari R. The role of telemedicine in hypertension management: focus on blood pressure telemonitoring. Curr Hypertens Rep 2015;17:535.

21 Parati G, Pellegrini D, Torlasco C. How digital health can be applied for preventing and managing hypertension. Curr Hypertens Rep 2019;21:40.

22 lellamo F, Sposato B, Volterrani M. Telemonitoring for the management of patients with heart failure. Card Fail Rev 2020;6:e07.

23 Chen C, Wang J, Pang L, et al. Telemonitor care helps CPAP compliance in patients with obstructive sleep apnea: a systemic review and meta-analysis of randomized controlled trials. Ther Adv Chronic Dis 2020;11:2040622320901625.

24 Franc S, Hanaire H, Benhamou P-Y, et al. DIABEO system combining a mobile APP software with and without Telemonitoring versus standard care: a randomized controlled trial in diabetes patients poorly controlled with a Basal-Bolus insulin regimen. Diabetes Technol Ther 2020;22:904-11.

25 Margolis KL, Asche SE, Dehmer SP, et al. Long-Term outcomes of the effects of home blood pressure Telemonitoring and pharmacist management on blood pressure among adults with uncontrolled hypertension: follow-up of a cluster randomized clinical trial. JAMA Netw Open 2018;1:e181617.

26 Omboni S, Gazzola T, Carabelli G, et al. Clinical usefulness and cost effectiveness of home blood pressure telemonitoring: meta-analysis of randomized controlled studies. J Hypertens 2013;31:455-67. discussion 467-458.

27 Bray EP, Jones MI, Banting M, et al. Performance and persistence of a blood pressure self-management intervention: telemonitoring and self-management in hypertension (TASMINH2) trial. J Hum Hypertens 2015;29:436-41.
28 Jones MI, Greenfield SM, Bray EP, et al. Patients' experiences of self-monitoring blood pressure and self-titration of medication: the TASMINH2 trial qualitative study. Br J Gen Pract 2012;62:e135-42.

29 McManus RJ, Mant J, Franssen M, et al. Efficacy of self-monitored blood pressure, with or without telemonitoring, for titration of antihypertensive medication (TASMINH4): an unmasked randomised controlled trial. Lancet 2018;391:949-59.

30 Monahan M, Jowett S, Nickless A, et al. Cost-Effectiveness of Telemonitoring and self-monitoring of blood pressure for antihypertensive titration in primary care (TASMINH4). Hypertension 2019;73:1231-9.

31 Duan Y, Xie Z, Dong F, et al. Effectiveness of home blood pressure telemonitoring: a systematic review and meta-analysis of randomised controlled studies. J Hum Hypertens 2017;31:427-37.

$32 \mathrm{He} \mathrm{T}$, Liu X, Li Y, et al. Remote home management for chronic kidney disease: a systematic review. J Telemed Telecare 2017;23:3-13.

33 Makhija D, Alscher MD, Becker S, et al. Remote monitoring of automated peritoneal dialysis patients: assessing clinical and economic value. Telemed J E Health 2018;24:315-23.

34 Luo L, Ye M, Tan J, et al. Telehealth for the management of blood pressure in patients with chronic kidney disease: a systematic review. J Telemed Telecare 2019;25:80-92.

35 Moher D. Shamseer L, Clarke M, et al. Preferred reporting items for systematic review and meta-analysis protocols (PRISMA-P) 2015 statement. Systematic Reviews. 2015;4(1):1.

36 Higgins JPT TJ, Chandler J, Cumpston M, et al. Cochrane Handbook for Systematic Reviews of Interventions version 6.0 (updated July 2019). Cochrane, 20192019.

37 Hoy D, Brooks $P$, Woolf $A$, et al. Assessing risk of bias in prevalence studies: modification of an existing tool and evidence of interrater agreement. J Clin Epidemiol 2012;65:934-9.

38 WorldBank. World bank open data, 2020. Available: http://data. worldbank.org/indicator 